\title{
Bound states in two spatial dimensions in the noncentral case
}

\section{Citation}

Martin, André, and Tai Tsun Wu. 2004. "Bound States in Two Spatial Dimensions in the Noncentral Case." Journal of Mathematical Physics 45 (3): 922-31. https:// doi.org/10.1063/1.1639956.

\section{Permanent link}

http://nrs.harvard.edu/urn-3:HUL.InstRepos:41555809

\section{Terms of Use}

This article was downloaded from Harvard University's DASH repository, and is made available under the terms and conditions applicable to Other Posted Material, as set forth at http:// nrs.harvard.edu/urn-3:HUL.InstRepos:dash.current.terms-of-use\#LAA

\section{Share Your Story}

The Harvard community has made this article openly available.

Please share how this access benefits you. Submit a story.

Accessibility 
CERN-TH/2003-235

\title{
BOUND STATES IN TWO SPATIAL DIMENSIONS IN THE NON-CENTRAL CASE
}

\author{
André MARTIN \\ Theoretical Physics Division, CERN \\ CH - 1211 Geneva 23, Switzerland \\ and \\ Laboratoire de Physique Théorique ENSLAPP \\ F - 74941 Annecy-le-Vieux, France \\ Tai Tsun WU ${ }^{1}$ \\ Gordon McKay Laboratory, Harvard University \\ Cambridge, MA 02138-2901, U.S.A. \\ and \\ Theoretical Physics Division, CERN \\ CH - 1211 Geneva 23, Switzerland
}

\begin{abstract}
We derive a bound on the total number of negative energy bound states in a potential in two spatial dimensions by using an adaptation of the Schwinger method to derive the BirmanSchwinger bound in three dimensions. Specifically, counting the number of bound states in a potential $g V$ for $g=1$ is replaced by counting the number of $g_{i}$ 's for which zero energy bound states exist, and then the kernel of the integral equation for the zero-energy wave functon is symmetrized. One of the keys of the solution is the replacement of an inhomogeneous integral equation by a homogeneous integral equation.
\end{abstract}

CERN-TH/2003-235

August 2003

\footnotetext{
${ }^{1}$ Work supported in part by the U.S. Department of Energy under Grant No. DE-FG02-84-ER40158
} 


\section{Introduction}

In a previous paper [1], K. Chadan, N.N. Khuri and ourselves (A.M. and T.T.W.) obtained a bound on the number of bound states in a two-dimensional central potential. This bound has the merit that, for a potential $g V$, the coupling constant dependence for large $g$ is optimal, i.e., the same as the one of the semiclassical estimate [2]. Previous work on the subject was done by Newton [3] and Seto [4]. We also obtained a bound for the non-central case, but only by using a rather brutal method which consists of replacing the potential by a central potential which is defined, after choosing a certain origin, by

$$
\begin{aligned}
V_{c}(r)= & \operatorname{Inf} V(\vec{x}) \\
& |\vec{x}|=r
\end{aligned}
$$

Because of the monotonicity of the energy levels with respect to the potential, putting $V_{c}$ in our formulae will give a bound for the potential $V$. However, for potentials with singularities outside the given origin, this may lead to no bound at all. Our attention has been attracted by the fact that in condensed matter physics problems exist, where counting the bound states on a surface may be useful [5], but where it is very unlikely that the potential will be central, even approximately.

In the present paper, we obtain a bound on the number of bound states in a non-central two-dimensional potential, using an adaptation of the Schwinger method to derive the BirmanSchwinger bound [6] in the three-dimensional case. The condition under which we obtain a bound is

$$
\iint d^{2} x d^{2} y|V(x)|(\ell n|x-y|)^{2}|V(y)|<\infty .
$$

This condition is non-linear, just like that of Birman and Schwinger, but we show in the Appendix that it follows from the linear conditions

$$
\begin{array}{cc}
\int d^{2} x & (\ln (2+|x|))^{2}|V(x)|<\infty \\
\text { and } & \\
\int d^{2} x & V_{R}(|\vec{x}|) \ell n^{-}|x|<\infty,
\end{array}
$$

where $V_{R}$ is the circular decreasing rearrangement of $|V|$ (for the definition of $V_{R}$, see the Appendix).

Condition (2) has already been proposed by Sabatier [7]. Condition (3) appears in a forthcoming work by N.N. Khuri, A. Martin, P. Sabatier and T.T. Wu, dealing with the scattering problem. It has the advantage of showing more clearly what kind of behaviour the potential is allowed to have at short and large distances.

The strategy of Schwinger consists of counting the number of zero-energy bound states for a potential $g V$ for $0<g<1$ instead of the actual number of negative energy bound states for $g=1$. In three dimensions these two numbers are equal. Indeed, let $0<g_{1}, g_{2}, \ldots g_{n}<1$ be the coupling constants for which we have zero-energy bound states. Each $g_{i}$ is the origin 
of a bound state trajectory in the $E-g$ plane, $E_{i}(g)$, with $E_{i}\left(g_{i}\right)=0$. These trajectories are monotonous decreasing:

$$
\frac{d E_{i}}{d g}=\int V \psi^{2} d^{n} x
$$

by the Feynman-Hellmann theorem, but

$$
g_{i} \int V \psi^{2} d^{n} x=E-\int|\nabla \psi|^{2} d^{n} x<0 \text { for } E<0
$$

This shows that the number of negative-energy bound states is exactly the same as the number of $g_{i}$ 's $<1$. At the crossing of any pair of trajectories there is no problem because of their monotonicity.

The same result holds in two dimensions with one modification: any attractive potential (i.e., $\int d^{2} x V<0$ ), has a bound state for arbitrarily small $g$, with a binding energy going to zero for $g \rightarrow 0$ like $-\exp -(C / g)$ [1]. At $E=0$ it disappears and is not included in Schwinger's accounting, so we have to add one unit.

Since we only want a bound on the number of bound states, we can always replace $V(y)$ by $-V^{-}(y)$

$$
\begin{aligned}
& V^{-}(y)=0 \text { for } V>0 \\
& V^{-}(y)=-V(y) \text { for } V \leq 0
\end{aligned}
$$

Using $-|V(y)|$ instead of $-V^{-}(y)$ gives a more crude bound.

It can be shown that the general solution of the zero-energy Schrödinger equation

$$
-\Delta \psi-V^{-} \psi=0
$$

in the equivalent integral form

$$
\psi(x)=C-\frac{1}{2 \pi} \int \ell n k_{0}|x-y| V^{-}(y) \psi(y) d^{2} y
$$

has a general asymptotic behaviour for $|x| \rightarrow \infty$

$$
\psi(x)-C \sim-\ell n k_{0}|x| \frac{1}{2 \pi} \int V^{-}(y) \psi(y) d^{2} y+o(1)
$$

under the condition

$$
\int V^{-}(y)(\ln (2+|y|))^{2} d^{2} y<\infty
$$

which follows from condition (1) as shown in the Appendix.

Zero-energy bound states are characterized by the fact that $\psi$ is bounded. Hence we get the necessary condition:

$$
\int V^{-}(y) \psi(y) d^{2} y=0
$$

Now we have two possibilities: 
- I. At infinity $\psi(\vec{x}) \rightarrow 0$ and hence $C=0$ and those bound states wave functions satisfy a homogeneous integral equation

$$
\psi_{i}(x)=-\frac{g_{i}}{2 \pi} \int d^{2} y \ln \left(k_{0}|x-y|\right) V^{-}(y) \psi_{i}(y)
$$

(notice that the scale factor $k_{0}$ disappears because of condition (11)).

This is what happens in the case of a central potential for a non-zero azimuthal angular momentum $m$.

- II. At infinity, $\psi(\vec{x}) \rightarrow C$, with $C \neq 0$. In this case, the bound state wave functions satisfy an inhomogeneous integral equation. This case has been described in Ref. [8], where it is shown that for a central potential in two dimensions, the $m=0$ phase shift has the universal behaviour

$$
\delta(k) \sim \frac{\pi}{2 \ell n k}, \text { for } k \rightarrow 0
$$

except if there is a zero-energy bound state of type II. Then

$$
\delta(k) \ell n k \rightarrow 0
$$

In Ref. [8], a much stronger result is stated. This much stronger result, however, holds only for a very rapidly decreasing potential.

\section{Counting Bound States in Case I}

Following Schwinger, we symmetrize the kernel of the integral equation:

$$
\phi_{i}(x)=g_{i} \int K(x, y) \phi_{i}(y) d^{2} y
$$

with

$$
\begin{aligned}
\phi_{i}(x) & =\sqrt{V^{-}(x)} \psi_{i}(x) \\
K(x, y) & =-\frac{1}{2 \pi} \sqrt{V^{-}(x)} \ln k_{0}|x-y| \sqrt{V^{-}(y)} .
\end{aligned}
$$

If $V^{-}(x)$ vanishes in some regions, it seems impossible to go back from $\phi_{i}$ to $\psi_{i}$. However, this can be remedied by defining

$$
V_{\epsilon}^{-}(x)=V^{-}(x)+\epsilon \exp -\mu|x|
$$

Since the bounds we shall get are continuous in $V$, we can take the limit $\epsilon \rightarrow 0$ at the end.

$K$ can be written as

$$
K=\Sigma \frac{1}{g_{i}}\left|\phi_{i}><\phi_{i}\right|+R
$$


$R$ is a sum over states which do not satisfy (11). The $\phi_{i}$ 's in themselves do not form a complete set. If we define $a$ by

$$
a(x)=\frac{\sqrt{V^{-}(x)}}{\sqrt{\int V^{-}(y) d^{2} y}}
$$

we have

$$
<a \mid \phi_{i}>=0
$$

from property (11), and naturally $<a|a\rangle=1$.

If we define $\hat{T r}$, a trace restricted to the $\phi_{i}$ 's, we have

$$
\hat{\operatorname{Tr}} K=\Sigma \frac{1}{g_{i}}>\sum_{g_{i} \leq 1} \frac{1}{g_{i}}>N_{I},
$$

$N_{I}$ being the number of bound states of type I. However, this trace turns out to be divergent because of the logarithmic singularity of the kernel (the same happened in Schwinger's original work!), and we follow Schwinger to iterate the integral equation (15):

$$
\phi_{i}(x)=g_{i}^{2} \int K(x, y) K(y, z) \phi_{i}(z) d^{2} y d^{2} z
$$

and then

$$
\hat{\operatorname{Tr}} K^{2}=\Sigma \frac{1}{g_{i}^{2}}>N_{I}
$$

Forgetting the "hat" on the trace still gives a bound because $K^{2}$ is a positive operator (contrary to $\mathrm{K}$ !), but this bound depends on the scale parameter $k_{0}$ entering in the logarithm. Among the missing states in $\hat{T r}$ is the state $\mid a>$, orthogonal to the $\phi_{i}$ 's, and this one should be removed from the complete trace. In this way, we get

$$
N_{I}<\operatorname{Tr} K^{2}-<a\left|K^{2}\right| a>
$$

or more explicitly

$$
\begin{aligned}
& N_{I}<\frac{1}{(2 \pi)^{2}} \int V^{-}(x)\left(\ln k_{0}|x-y|\right)^{2} V^{-}(y) d^{2} x d^{2} y \\
& -\quad \frac{1}{(2 \pi)^{2}} \frac{1}{\int V^{-}(x) d^{2} x} \int V^{-}(x)\left(\ln k_{0}|x-z|\right) V^{-}(z)\left(\ln k_{0}|z-y|\right) V^{-}(y) d^{2} x d^{2} y d^{2} z
\end{aligned}
$$

It is visible that the second term is negative as we announced.

Rewriting $N_{I}$ as

$$
\frac{1}{\int V^{-}(z) d^{2} z} \int d^{2} x d^{2} y d^{2} z V^{-}(x) V^{-}(y) V^{-}(z)\left[\left(\ln k_{0}|x-y|\right)^{2}-\ln k_{0}|x-z| \ln k_{0}|y-z|\right]
$$

we see that (22) is manifestly independent of the scale factor $k_{0}$. 


\section{Counting Bound States in Case II}

At first it would seem that Schwinger's technique will not work because, in Eq. (8), the constant is not zero and therefore we deal with an inhomogeneous integral equation which can be written, after the same changes of variables as in Section 2, given by (16) and (19):

$$
\phi_{i}=C_{i} a+g_{i} K \phi_{i}
$$

with, again,

$$
<a \mid \phi_{i}>=0 \text { and }<a \mid a>=1
$$

Equation (24) is precisely the key property which will make it possible to replace (23) by a homogeneous equation.

Again, the $\phi_{i}$ 's corresponding to different $g_{i}$ 's are orthogonal because

$$
\begin{aligned}
<\phi_{i} \mid \phi_{j}> & =C_{j}<\phi_{i}\left|a>+g_{j}<\phi_{i}\right| C \phi_{j}> \\
& =C_{j}<\phi_{j}\left|a>+g_{i}<\phi_{i}\right| C \phi_{j}>.
\end{aligned}
$$

Hence, from (24):

$$
\left(\frac{1}{g_{i}}-\frac{1}{g_{j}}\right)<\phi_{i} \mid \phi_{j}>=0
$$

Let us call $\mathcal{S}$ the Hilbert space associated to the integral equation (23), and construct a new Hilbert space by removing the element $a$ :

$$
\mathcal{S}=\mathcal{S}^{\prime} \oplus\{a\}
$$

We want to define a new operator $K^{\prime}$ acting in $\mathcal{S}^{\prime}$. Let

$$
b=K a .
$$

Notice that

$$
<b\left|\phi_{i}>=-\frac{C_{i}}{g_{i}}<a\right| a>=-\frac{C_{i}}{g_{i}} .
$$

We try

$$
K^{\prime}=K-|b><a|-|a><b|+C|a><a|
$$

where $C$ will be chosen so that

$$
K^{\prime} a=0 .
$$

We have

$$
K^{\prime}|a>=| b>-|b>-<b| a>|a>+C| a>,
$$

and hence we take

$$
C=<b|a>=<a| K \mid a>
$$


$K^{\prime}$ is Hermitian like $K$, and we get

$$
\begin{aligned}
g_{i} K^{\prime} \mid \phi_{i}> & =g_{i}|K| \phi_{i}>-g_{i}<b\left|\phi_{i}>\right| a> \\
& =g_{i}|K| \phi_{i}>+C_{i} \mid a>.
\end{aligned}
$$

Hence

$$
g_{i} K^{\prime}\left|\phi_{i}>=\right| \phi_{i}>
$$

which is homogeneous.

To get a bound on the number of bound states of type II, we have to get a bound on trace $K^{\prime 2}$ (not surprisingly, trace $K^{\prime}$ is divergent). It is a lengthy but straightforward exercise to calculate that trace, which gives

$$
N_{I I}<\operatorname{tr} K^{2}-2<a\left|K^{2}\right| a>+(<a|K| a>)^{2}
$$

The last two terms give an overall negative contribution. The first term is the same as the one appearing in $N_{I}$. It is easy to see that the right-hand side of (32) is independent of the scale parameter $k_{0}$ entering into the kernel $K$. Finally, let us notice that the treatment of case II contains case I because, in the argument, it has never been said that $C_{i} \neq 0$. Equation (31) holds irrespective of whether $C_{i}=0$ or $C_{i} \neq 0$. Notice that the bound on $N_{I}$ is larger than the bound on $N_{I I}$. Therefore the bound on $N_{I}$ becomes completely obsolete.

\section{Concluding Remarks}

If we include the bound state with evanescent energy for zero coupling constant, we get the bound

$$
N<1+\operatorname{tr} K^{2}-2<a\left|K^{2}\right| a>+(<a|K| a>)^{2} .
$$

Dropping the last two terms still gives a scale-dependent bound - which can be minimized with respect to the scale - which precisely appears in condition (2), itself following from the linear condition (3) as shown in the Appendix.

Conditions (2) and (3) both allow a potential behaving like

$$
\frac{1}{r^{2}(\ln r)^{3+\epsilon}}
$$

at infinity, with local singularities not worse than

$$
-\frac{1}{\left|r-r_{0}\right|^{2}\left(|\ell n| r-r_{0}||\right)^{2+\epsilon}},
$$

$\epsilon$ positive, arbitrarily small. Both conditions are violated for $\epsilon<0$. However, we shall see in the Appendix that (2) is definitely weaker than (3).

Our bound has the merit of being valid for the non-central case, which, as we said in the Introduction, is important for solid-state physics. However, for a potential $g V$, it behaves like 
$g^{2}$ for large $g$, while in Ref. [1], in the central case, we get a bound behaving like $g$. In Ref. [1] we make a conjecture which is very far from being proved, but clever mathematical physicists might prove it or something similar. The present work should be considered only as a first step which could possibly give reasonable results for not too large $g$.

\section{Acknowledgements}

We are grateful to P. Sabatier for suggesting the use of the non-linear expression defined by (2) for potentials in two dimensions. Our work was stimulated by discussions with our colleagues K. Chadan and N.N. Khuri. One of us (T.T.W.) would also like to thank the CERN Theoretical Physics Division for hospitality. This paper was put in final form while one of us (A.M.) was visiting the "Institut des Hautes Etudes Scientifiques", whose hospitality is acknowledged.

\section{Appendix: Comparison of condition (2) and condition (3)}

Condition (2) is

$$
I=\int d^{2} x d^{2} y V^{-}(x)(\ln |x-y|)^{2} V^{-}(y)<\infty
$$

Condition (3) is a set of two conditions:

$$
\begin{gathered}
\int d^{2} x(\ln (2+|x|))^{2} V^{-}(x)<\infty \\
\int d^{2} x V_{R}(|x|) \ell n^{-}(|x|)<\infty .
\end{gathered}
$$

In (A.3) we use:

$$
\begin{aligned}
-\ln ^{-}(|x|) & =0 \text { if }|x|>1 \\
& =-\ln |x| \text { if }|x|<1
\end{aligned}
$$

$-V_{R}(|x|)$, the circular decreasing rearrangement of $V^{-}(x)$. Since this notion is not very well known among physicists, let us remind the reader that $V_{R}(|x|)$ is a decreasing function of $|x|$, such that

$$
\mu\left(V_{R}(|x|)>A\right)=\mu\left(V^{-}(x)>A\right), \forall A,
$$

where $\mu$ is the Lebesque measure. In more familiar terms, the rearranged Mont Blanc would be a mountain with axial symmetry, with a single peak, such that the surface between the level lines would be the same as the surface between the level lines of the original Mont Blanc (rather awfully dull!).

We shall prove first that the convergence of $I$ in (A.1) follows from the convergence of (A.2) and (A.3). More exactly, we shall get an explicit bound on (A.1) in terms of (A.2) and (A.3). We write

$$
I=I_{+}+I_{-}
$$


with

$$
\begin{aligned}
& I_{+}=\int d^{2} x d^{2} y V^{-}(x)\left(\ell n^{+}|x-y|\right)^{2} V^{-}(y) \\
& I_{-}=\int d^{2} x d^{2} y V^{-}(x)\left(\ell n^{-}|x-y|\right)^{2} V^{-}(y)
\end{aligned}
$$

$\ell n^{-}$has already been defined. $\ell n^{+}(a)=\ell n a$ for $a \geq 1,=0$ for $a<1$. It is elementary to get a bound on $I_{+}$from (A.2) only. Indeed,

$$
0<\ln ^{+}|x-y|<\ln ^{+}(|x|+|y|)<\ln (2+|x|)+\ln (2+|y|)
$$

and thus

$$
\left(\ell n^{+}|x-y|\right)^{2}<2\left[(\ell n 2+|x|)^{2}+(\ln (2+|y|))^{2}\right]
$$

Hence

$$
I_{+}<4 \int d^{2} x V^{-}(x) \int d^{2} y V^{-}(y)(\ln (2+|y|))^{2} .
$$

The convergence of the right-hand side of (A.8) follows directly from (A.2).

Concerning $I_{-}$, we use a rearrangement inequality due to Luttinger and Friedberg [9], which says

$$
\iint A(x) B(|x-y|) C(y) d^{2} x d^{2} y \leq \int A_{R}(|x|) B_{R}(|x-y|) C_{R}(|y|) d^{2} x d^{2} y
$$

where $A, B, C$ are non-negative functions and $A_{R}, B_{R}, C_{R}$ are their decreasing rearrangements. Since $\ell n^{-}$and $\left(\ell n^{-}\right)^{2}$ are decreasing functions of their argument they are their own rearrangement. Hence

$$
I_{-}<\int d^{2} x d^{2} y V_{R}(|x|)\left(\ell n^{-}(|x-y|)\right)^{2} V_{R}(|y|)
$$

In (A.10), we can carry out first the angular integration, the angle $(\vec{x}, \vec{y})$ appearing only in $\ell n^{-}$. However, to be able to do that easily we have to sacrifice some information, i.e., use $\left(\ell^{-}|x-y|\right)^{2} \leq(\ln (|x-y|))^{2}$. We have to calculate

$$
\int \frac{d \theta}{2 \pi}\left(\ln \left(|x|^{2}+|y|^{2}-2|x||y| \cos \theta\right)\right)^{2}
$$

We have

$$
\ln \left(|x|^{2}+|y|^{2}-2|x||y| \cos \theta\right)=\ln \left(|x|-|y| e^{i \theta}\right)+\ln \left(|x|-|y| e^{-i \theta}\right) .
$$

Assume $|x|>|y|$. Then we get

$$
\ln \left(|x|^{2}+|y|^{2}-2|x||y| \cos \theta\right)=2\left[\ln |x|-\Sigma\left(\frac{|y|}{|x|}\right)^{n} \frac{\cos n \theta}{n}\right]
$$

Hence, if $|x|>|y|$, using the orthogonality of the $\cos n \theta$ :

$$
\int \frac{d \theta}{2 \pi}\left(\ln \left(|x|^{2}+|y|^{2}-2|x||y| \cos \theta\right)\right)^{2}=4(\ln |x|)^{2}+2 \sum_{n=1}^{\infty}\left(\frac{|y|}{|x|}\right)^{2 n} \frac{1}{n^{2}}
$$


We see a dilogarithm, or Spence function, appearing on the right-hand side. However, we only need to notice that

$$
\sup _{|y| \leq|x|} \int \frac{d \theta}{2 \pi}\left(\ln |x|^{2}+|y|^{2}-2|y||x| \cos \theta\right)^{2}=4(\ln |x|)^{2}+2 \sum_{n=1}^{\infty} \frac{1}{n^{2}}=4(\ln |x|)^{2}+\frac{\pi^{2}}{3} .
$$

In this way we get

$$
I_{-}<(2 \pi)^{2} \times 2 \int_{|x|>|y|}|x| d|x||y| d|y| V_{R}(|x|) V_{R}(|y|)\left[4(\ln |x|)^{2}+\frac{\pi^{2}}{3}\right]
$$

Again, we split the integral into

$$
\begin{aligned}
& 32 \pi^{2} \int_{|x|>|y|}|x| d|x||y| d|y| V_{R}(|x|) V_{R}(|y|)\left(\ell n^{-}|x|\right)^{2} \\
& +32 \pi^{2} \int_{|x|>|y|}|x| d|x||y| d|y| V_{R}(|x|) V_{R}(|y|)\left(\ell n^{+}|x|\right)^{2} \\
& +\frac{4 \pi^{4}}{3} \quad\left[\int|x| d|x| V_{R}(|x|)\right]^{2}
\end{aligned}
$$

In the first term of (A.13) we can replace $\left(\ell n^{-}(|x|)\right)^{2}$ by $\ell n^{-}|x| \ell n^{-}|y|$, since $|x|>|y|$ and since $\ell n^{-}$is decreasing.

In the second term, we can drop the restriction $|x|>|y|$ and notice that

$$
\int d^{2} x V_{R}(|x|)\left(\ell n^{+}(|x|)\right)^{2}<\int d^{2} x V_{-}(x)\left(\ell n^{+}(|x|)\right)^{2} .
$$

Indeed, $\int d^{2} x A_{R}(x) \phi(|x|)$, where $\phi(|x|)$ is increasing, is less than $\int d^{2} x A(x) \phi(|x|)$. Suppose that $\phi(|x|) \rightarrow L$. Then

$$
\int d^{2} x A_{R}(x) \phi(|x|)=\int d^{2} x A_{R}(x) L-\int d^{2} x A_{R}(x)(L-\phi(|x|)) .
$$

$L-\phi(|x|)$ is its own decreasing rearrangement and following the well-known properties

$$
\int A_{R} B_{R} d^{2} x \geq \int A(x) B(x) d^{2} x
$$

and

$$
\int A_{R}(|x|) d^{2} x=\int A(x) d^{2} x
$$

we get the desired property. If $L$ is infinite, we can use a limiting procedure. Finally, we get

$$
\begin{aligned}
I_{-} & <16 \pi^{2}\left[\int d^{2} x V_{R}(|x|) \ell n^{-}|x|\right]^{2} \\
& +32 \pi^{2} \int d^{2} x V_{-}(x) \int d^{2} y V_{-}(y)(\ln (2+|y|))^{2} \\
& +\frac{4 \pi^{4}}{3}\left[\int d^{2} x V_{-}(x)\right]^{2}
\end{aligned}
$$


From (A.2) and (A.3) we see that $I_{-}$is bounded. This concludes the proof.

One question is: can we go in the opposite direction? Assume that we know that (A.1) holds. There exists certainly a region $\left|x-x_{o}\right|<R$ where $\operatorname{Inf} V^{-}=m>0$. If such a region did not exist, $V^{-}$would be zero almost everywhere! So

$$
I>\pi R^{2} m \int_{|x|>\left|x_{o}\right|+R+2} V^{-}(x) \ln [2+|x|]^{2} d^{2} x
$$

Now we choose $y_{o}>4+\left|x_{o}\right|+R+2$ such that

$$
\operatorname{Inf}_{\left|y-y_{o}\right|<R^{\prime}} V_{-}(y)=m^{\prime}>0,
$$

then

$$
I>\pi R^{\prime 2} m^{\prime} \int_{|x|<\left|x_{o}\right|+R+2} V^{-}(x)\left(\ln \left(4-R^{\prime}\right)\right)^{2} d^{2} x .
$$

This proves that the convergence of (A.1) implies the convergence of (A.2).

It is not possible to deduce (A.3) from (A.1) because (A.3) involves $V_{R}$ and (A.1) does not. However, in practice the conditions are very similar. Nevertheless, the following example shows that (A.3) is stronger than (A.1), even for a potential which does not need rearrangement: take the central potential

$$
\begin{aligned}
V(|x|) & =-\frac{1}{|x|^{2}|\ln x|^{2}(\ln |\ln | x||)^{\gamma}} \text { for }|x|<\frac{1}{2 e}, \\
& =0 \text { for }|x| \geq \frac{1}{2 e} .
\end{aligned}
$$

For $\gamma \leq \frac{1}{2}$ (A.1) and (A.3) are divergent, for $\frac{1}{2}<\gamma \leq 1$ (A.1) is convergent and (A.3) is divergent, for $\gamma>1$ (A.1) and (A.3) are convergent.

\section{References}

[1] K. Chadan, N.N. Khuri, A. Martin and T.T. Wu, J. Math. Phys. 44 (2003) 406.

[2] A. Martin, Helv.Phys.Acta 45 (1972) 140, H. Tamura, Proc. Jpn. Acad. 50 (1974) 19.

[3] R.G. Newton, J. Math. Phys. 3 (1962) 867, J. Operator Theory 10 (1983) 119.

[4] N. Seto, Publ. RIMS, Kyoto University 9179) 429.

[5] F. Bassani, T. Martin, private communications.

[6] J. Schwinger, Proc. Nat. Acad. Sci. USA 47 (1961) 122;

M. Birman, Math. Sb. 55 (1961) 124;

English translation Amer. Math. Soc. trans. 53 (1966) 23. See also:

M.S. Birman, Dokl. Acad. Nauk. SSSR, 129 (1959) 239 (in Russian. No English translation available.) 
[7] P. Sabatier, private communication.

[8] K. Chadan, N.N. Khuri, A. Martin and T.T. Wu, Phys. Rev. D58 (1998) 025014.

[9] J.M. Luttinger and R. Friedberg, quoted in:

J.M. Luttinger, J. Math. Phys. 14 (1973) 1450. 